\title{
Congestion Control in MANET using Modify AODV with IRED Congestion Control Algorithm
}

\author{
Gurmeen Kaur \\ Research Scholar at \\ Department of Computer \\ Science \& Engg. \\ Sachdeva Engg. College for \\ Girls, Gharuan
}

\author{
Harinder Kaur \\ Assistant Professor at \\ Department of Computer \\ Science \& Engg. \\ Sachdeva Engg. College for \\ Girls, Gharuan
}

\author{
Rakesh Kumar \\ Assistant Professor at \\ Department of Computer \\ Science \& Engg. \\ Sachdeva Engg. College for \\ Girls, Gharuan
}

\begin{abstract}
MANET is a field of networking that used for sharing of information between different links. In this process data has been transmitted from different devices to a single device to forward or store valuable information. Due to transmission of data from multiple sources to a single node occur congestion problem in the network. In this paper an approach has been proposed for congestion avoidance in MANET. This approach based on queue fulfillment prediction to avoid congestion and path management for data transmission. This approach provides better performance than previous proposed approaches.
\end{abstract}

\section{Keywords}

MANET, Congestion, Reactive, RED and IRED

\section{INTRODUCTION}

The routing protocols are design and analysis these types of issues in MANET. It is the main essential

Objective of a MANET directing convention is to create a correct and capable route between couples of two hosts for using the transmission messages.

Frequently different directing conventions [2, 3] have been proposed for MANETS. We can be classified into the two classified table. These are determined and on the interest.

The table-driven direction-finding conventions are like and come as a features development of those for the wired systems together with Internet. They fundamentally develop proactive plans, which attempt to keep up conventional cutting-edge directing data from every one host to all other hub in the MANET.

These type of conventions require each one host to keep up one or extra tables to contain most current direction-finding data, and several change in system topology desires to be reflected by TV redesigns data all from beginning to end the system with a particular end goal to keep up an expected system view.

Another time, the on-interest direction-finding conventions take a relaxed methodology to directing.

All the motivation following the on-interest conventions is to lessen huge evaluate of transparency for keeping up the direction-finding the table, in the table-driven conventions in the element MANET.

It is the source launched plans which don't up alternately for all time repairs their route tables with latest route topology. The main directing makes route just as wanted by the source hub. At this point while a hub obliges a route to a terminus, its starts a route exposure transform inside the system. This methodology is used to finish once one or more courses are exposed or all conceivable route stages have been analyzed. however, direction-finding transparency for on-interest conventions might be still considerable for the most part in light of the fact that is the flooding methodology utilized as a part of finding courses, wherever the source (i.e., the host looking for a course) floods the whole system with an analysis parcel in looking a route to the finishing point [4].

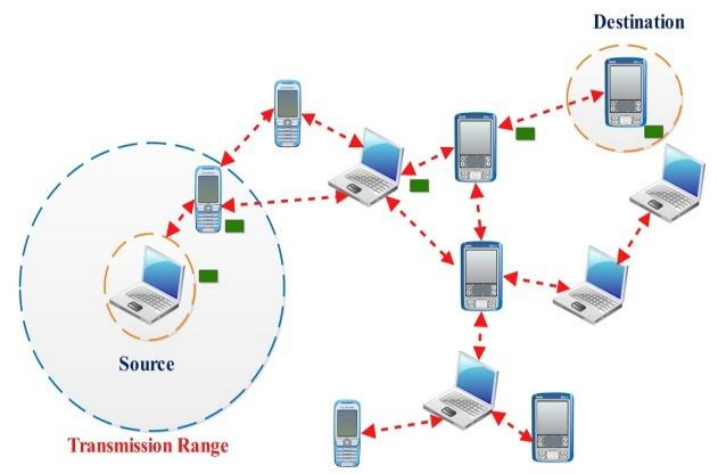

Fig 1 MANET Architecture

Dynamic source routing (DSR) it is the dynamic source routing gathering is one of the every one additional for the mainly part recognized on the demand directing conventions. It is the universal to believe the DSR convention with the multiple courses because they might be manufactured along with the route revelation by flooding. The Dynamic Source Routing (DSR) gathering proposed in furthermore has a choice of keeping up a variety of courses; consequently an alternate course can be utilized upon dissatisfaction of the necessary one. Subsist that since it may in DSR, an excess of courses are kept up in an insignificant way, exclusive of any respect to their definitive helpfulness. In the execution analysis of DSR conventions has not been aimed at in [5].

\subsection{Security Goals}

Availability: in the Accessibility is implies the benefits are open to approved gatherings at fitting times. The Accessibility applies equally to in sequence and to administrations. It is guarantees the survivability of system administration apart from refusal of administration assault.

Confidentiality: Confidentiality called it is guarantees to computer connected resources are gotten to just by accepted gatherings. That is, just the individuals who consideration to have admittance to impressive will in fact get that get to. To keep up secrecy of some classified data, we have to keep them 
mystery from every element that does not have profit to get to them. The Secrecy is commonly called secrecy or safety [6].

Integrity: the integrity is reliability implies that benefits can be changed presently by accepted gatherings and now in accepted way. Transform incorporates composing, developing status, erasing and manufacture. Constancy guarantees that a communication being exchanged is never defiled.

Authentication: it is the Confirmation empowers a hub to guarantee the behavior of associate hub it is the equivalent with. Substantiation is basically guarantee that members in association are confirmed and not impersonators. The Validness is guaranteed in light of the fact that just the accurate blue sender can construct a message that will decode reasonably with the common key [7].

Authorization: the authorization is property relegates various access rights to varied sorts of clients. Used for occurrence a system management can be performed by system administrator just.

\section{REVIEW OF LITERATURE}

Jhuria M. in this paper author discuss with mobile ad hoc network is picking up notoriety particularly for the applications where the anxiety of system basis is unreasonable like military application, Fiasco administration and remote sensing. Despite the fact that the MANET gives an unusual method for communication item MIN: without system structure though it services a few downsides and limits (chiefly in course finding and support) which probable to be corrected. This is the theory displays an adaptable specialist's based for system to improve the accomplishment of the DSR direction-finding congregation utilizing portable operators. The element source directing reunion is a directly forward and industrious direction-finding convention outlined predominantly

For develop in multi-jump remote particularly appointed systems of moveable hubs. The DSR authorization the system to be totally dealing with toward oneself or chest rating toward oneself, without the necessity for any subsisting system base or administration.

The convention is as one of the two systems of the conference and route protecting, which assist to allow hubs to find and keep up resource courses and stay resource courses to subjective objectives in the unsuspecting system. The operation of source direction-finding grant bundle directing to be critical circle free stays away from the condition for stateof-the-art direction-finding data.

\section{The adaptable specialists is basically a system which}

Development by the home machine and behind that it spreads through the scheme hubs as well as sends reverse the satisfying data. In this paper the proposed method is tried by mimicking a portable unprepared system utilizing system experiment system and the results demonstrate that it upgrades the execution of the system.

Ahmad S. "Performance Analysis of DSR \& Extended DSR Protocols", in this paper particularly agreed system is congregation of remote hubs to build a system without any recognized base or incorporated direction/management. In this such as system, topology changes increasingly and because of restrictions of transfer speed, transmission range and force directing turns into a necessary issue. A ton of exertion has been accepted out infield of direction-finding in impromptu system following 1990. The Element Source Routing convention (DSR) gives essential and productive directing for multi hop specially appointed system of portable hubs. In This paper exhibits an activity based implementation assessment and association between usual DSR and amplified DSR. It is uses an extraordinarily planned structure which expands on the Global Mobile Information System Simulator (Glomosim).A few enhancements of DSR have been as of now been executed in Glomosim. A few distinguishing reproduction results reveal that execution showed signs of development by conventional (officially executed) DSR.

Shen Ming-yu "Research and Analysis on Secure DSR Routing Protocol Based on Strand Space", this paper describes the confirmation Test Theory of strand space initially and the theory is finished since of the demands of mobile ad hoc network routing procedure safety analysis. By analyzing the reachable security DSR routing protocol leaks, author a new Ariadne-S set of rules model is proposed based on Ariadne routing protocol. Finally it is proved that the recurring routing in sequence from the process of routing finding is secure and believable by using strand space model.

\section{Problem Formulation}

Mobile ad-hoc network is branch of networking that deals with communication between nodes without interference of any external device. In MANET various node have been used for communication from source to destination. Source node broadcast a request message over the network for data transmission. Intermediate nodes receives request and discover a route for data transmission and reply to source with all route information.

In MANET various issues have been encountered that degrades the performance of the network. These problem are congestion occurs in the network sue to peer to peer communication. Sometime a single node received large amount data than that a node can transmit further to destination. This issue get extend to data loss due to congestion occurred on a node. In MANET route discovery mechanism has been utilized for path generation from source to destination so that optimum path can be selected. Optimum path assures guaranteed delivery of data from source to destination. In MANET various algorithm had been purposed for congestion avoidance but these algorithm are too complex that consumes too much time and increase network overload.

In the purposed work enhanced IRED has been used with new route discovery mechanism for avoidance of above defined problems in MANET. IRED has been enhanced on the basis of weight age factor provided in the algorithm with queue length.

\section{METHDOLOGY}

MANET is extensions of mobile network that utilize external source for data transmission from one node to another. In mobile ad-hoc network data has been transmitted over the network with utilization of any external source. In this process of data transmission from source to destination intermediate node have been utilized for message forwarding. In this process various phases have to be followed that have been described in flow work of the purposed system. 


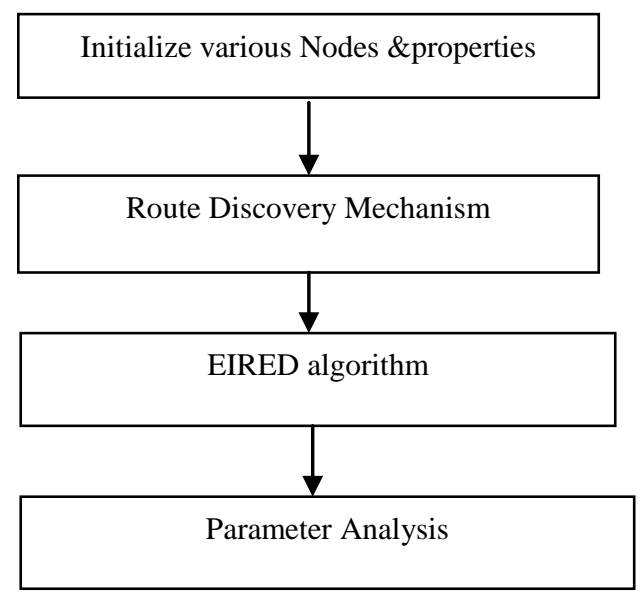

Fig 2 Flow Diagram for Purposed Work

This figure represents flow of the purposed work that has been used for MANET congestion avoidance. Congestion is a major issue in MANET due to multiple source and destinations available in the network.

\section{- Generation of MANET}

MANET has been developed by localization of Nodes in the particular area and these nodes have been provided a location in the area and mobility model has been defined for mobility of the nodes. These nodes have been configured by defining routing protocol, antenna, MAC type, Queue Type, Queue Length and link layer configuration. On the basis of these parameters nodes have been initialized and used for transmission of information form source to destination.

\section{- Route Discovery Process}

In MANET data has been forwarded from source to destination using intermediate nodes with the help of routing protocols. Due to mobility in MANET reactive routing protocols have been used so that path can be changed dynamically for data transmission. In this process source node broadcast a route request message and node in the transmission range receives the request and match the destination id and if destination ID is matched then route reply message has been revert back to source else this node forward RREQ to other one-hop neighbor nodes. Other nodes matched ID until a path is not available to transmit data.

\section{- Congestion Avoidance}

Congestion is a major issue in wireless networks due to nodes receives more data than that it can forward from multiple links. This cause queue length provided in the network gets occupied by waiting packets that prone to dropping of all the packets incoming from other sources. In the process of congestion control enhanced IRED algorithm has been purposed that utilize minimum and maximum threshold and weightage priority to different paths for data transmission in the purposed work. On the basis of this approach congestion in MANET has been avoided so that delay can be decreased and throughput of the system can be increased.

\section{Step 1}

Initialization of variable for Router buffer

If (Crz> Threshold), (Communication between nodes not possible)

Else If ( $\mathrm{Rz}<=$ Threshold $)$

(Allow to communicate and transmitting data)

\section{Step 2}

Compute average queue size (avg) to evaluate whether or not to drop the packet.

$\operatorname{Avg}=(1-W q)$ avg $+W q * q$

$\mathrm{Pa}=\mathrm{Pb}(1 /(1$-count $* \mathrm{~Pb})$

$\mathrm{Pb}=\operatorname{maxp}[($ avg- minth $) /($ Maxthreshold-minthreshold $)$ count minq and maxq

Step3

The source node first checks whether a node is in range of the router

If (dis <=Ran)

(Then only allow source to send packet to the destination)

Else if (dis > Ran)

(Then message can't be transmitted)

Step4

Now if node comes under the range of router.

Avg $<-0$ and count $<--1$

//Initialization of variable (For each packet arrival)

If (the queue is non-empty)

$\{$ Avg $=(1-W q)$ avg $+W q * q\}$

Else if (avg>Maxth)

\{Else if (maxth <queue_size)

(Select a path having highest weightage to transmit data)

$\{$ Maxth $=$ maxth $+1 ; \mathrm{pa}=\mathrm{Pb} ;\}$

Else $\mathrm{Pa}=1 ;\}\}$

Step5

Else if (month $\leq$ avg $<$ maxth)

$\{$ If (thdif $>=1 *$ queue_size)

$\{$ Mid $=(\operatorname{maxth}+\operatorname{minth}) / 2$;

If (avg <mid)

\{Maxth -1 and minth $+1 ; \mathrm{Pb}=\mathrm{pa} ;\}$

Increment count with probability pa and

Mark the arriving packet count $<-0\}$

Else count <- -1

When queue become empty

Qt<- time

Step6

Else (Avg <= Minth)

(Compute various possible paths to transmit data)

\{If (Thdif $>=1 *$ Queue_size)

$\{$ Maxth $=$ Maxth $-1 ; \mathrm{Pb}=\mathrm{pa} ;\}$

Else $\mathrm{Pa}=0$;

Notations - The following notations are used in IRED Algorithm. Ran=Range, dis= distance of the nodes from the router, $\mathrm{qt}=$ start of the queue idle time, $\mathrm{Crz}=$ critical zone, $\mathrm{Rz}=$ Radio zone, avgAverage Queue Size, Wq=Weight Parameter, $0<\mathrm{Wq}<=1, \mathrm{q}=$ Current queue size, $\mathrm{Pb}=$ Immediate marking probability, $\mathrm{vPa}=$ Accumulative drop, probability, Maxp=Maximum value of $\mathrm{Pb}$

On the basis of this approach congestion in MANET has been decreased so the network efficiency can be increased to an extent.

\section{RESULTS}

In the purposed work MANET has been designed by initializing nodes and mobility to nodes. Various source and receivers have been defined in the network to transmit information. In this process nodes have been deployed in the particular area for transmission of data to different destinations.

In the purposed work various input parameters have been defined for simulation of purposed work. On the basis of these parameters different simulation in MANET has been performed to enhance performance of the network. These simulation parameters are essential for simulation setup of the MANET 
Table 1 Simulation parameter setup

\begin{tabular}{|l|l|}
\hline Parameter & Description \\
\hline Number of nodes & 50 \\
\hline Antenna Type & Omni \\
\hline Area & $1000 * 1000$ \\
\hline MAC type & 802.11 \\
\hline Queue Type & Drop-tail \\
\hline Queue Length & 50 \\
\hline Traffic Type & CBR \\
\hline Mobility Model & Random Way-Point \\
\hline Routing Protocol & Reactive \\
\hline Packet Size & 128,256 bits \\
\hline ulation Time & $100 \mathrm{sec}$ \\
\hline
\end{tabular}

In the purposed model simulation has been done to transmit message from source using intermediate nodes to destination. This simulation has been performed for analysis of various parameters to avoid congestion occurrence in the network. Various parameters have been analyzed for performance evaluation of purposed work

\section{a. Packet Delivery Ratio}

Packet delivery ratio has been measured for efficiency evaluation of purposed model. In this ratio between total numbers of packet properly delivered has been measure to total number of packets transmitted.

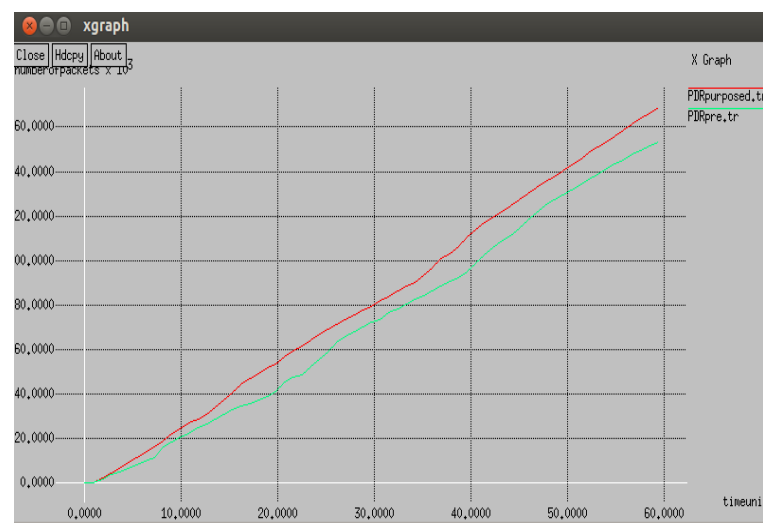

Fig 3 Packet Delivery Ratio

\section{b. Throughput}

Throughput has been measured for effective efficiency to transmit number of bits by the network. It is computed by ratio between total number of bits successfully transmit to per unit of time.

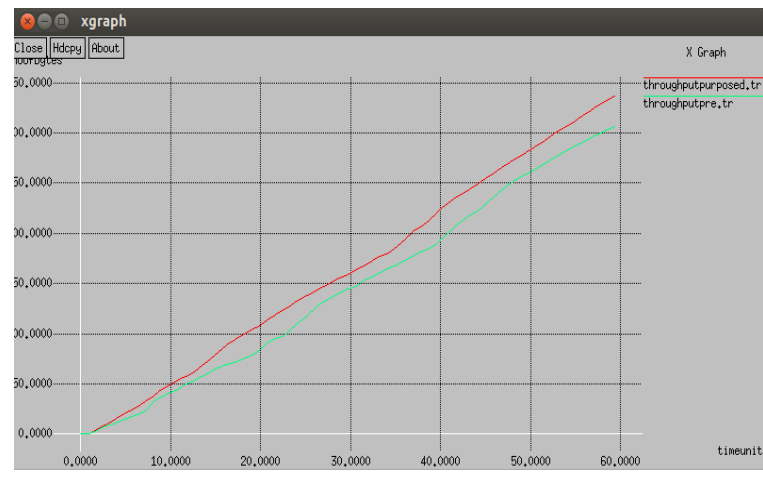

Fig 4 throughput

\section{c. Congestion}

Congestion is measured to check overall performance of the network. In the purposed work congestion window that occurred in the network has been measured.

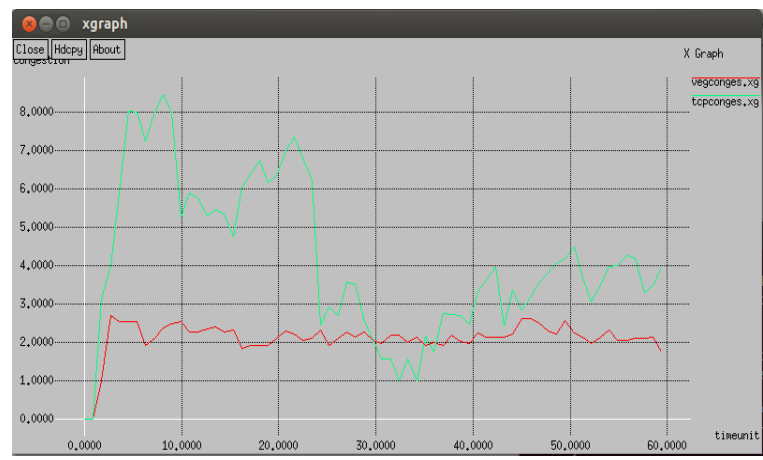

Fig 5 Congestion window Size

On the basis of these parameters purposed approach provide better efficiency than previous approaches.

\section{CONCLUSION\& FUTURE SCOPE}

MANET is used for transmission of data in secure way without interference of any external device. In this paper a new approach has been purposed for selection of optimal path on the basis of weightage to avoid congestion in the network. This approach use queue length and weightage factor to computes best route or changes to be made in the route if queue length has been approximate near to full then route has been neglected and new route on the basis of weightage have to be followed. By analyzing various parameters for performance evaluation we can conclude that purposed approach provide better results.

\section{REFERENCES}

[1] Shivashankar "Designing Energy Routing Protocol With Power Consumption Optimization in MANET", IEEE Conf. on MANET 2014, pp. 192-197.

[2] Dong-Li Zhang, Wen-cheng Jiao, Zheng Jian-Ling "Research and improvement of DSR protocol in Ad Hoc Network", Industrial and Information Systems (IIS), vol.1, pp. 242-244, IEEE, 2010.

[3] Zhiyong Shi, Shenquan Zhu, Zhenyu Zhang "Study on application of DSR protocol to mobile communication system", Mobile Technology, Applications and Systems, pp.-5, IEEE, 2005.

[4] Varshney, "Performance improvement of MANET under DSR protocol using swarm optimization", Issues and Challenges in Intelligent Computing Techniques (ICICT), PP. 58-63, IEEE, 2014.

[5] Jhuria, M., Singh, S. "Improve Performance DSR Protocol by Application of Mobile Agent", Communication Systems and Network Technologies (CSNT), pp. 336-340, IEEE, 2014.

[6] Ahmad, S, Aswan, I., Waqqas, A., Ahmad, B. "Performance Analysis of DSR \& Extended DSR Protocols", Modeling \& Simulation, pp. 191-196, IEEE, 2008.

[7] Shen Ming-yu, Li Cang-yuan, "Research and Analysis on Secure DSR Routing Protocol Based on Strand Space", Electrical and Control Engineering (ICECE), pp. 2917 2920, IEEE, 2010.

[8] J. Broch, D. Johnson and D. Maltz, "Dynamic Source 
Routing in Wireless Ad Hoc Networks," in Mobile Computing, eds. T. Imielinski and H. Korth (Kluwer Academic, 1996) Chapter 5.

[9] Yu-Liang Chang, Ching-Chi Hsu, "Connection-Oriented Routing in Ad Hoc Networks Based on Dynamic Group Infrastructure," it Proc. of the Fifth IEEE Symposium on Computers and Communications (ISCC 2000), pp. 587593, July 04 - 06, 2000 Antibes, France

[10] Ming-Hong Jiang, Rong-Hong Jan, "Ancient Multiple Paths Routing Protocol for Ad-hoc Net- works," The 15th International Conference on Information
Networking (ICOIN'01), January 31 - February02, 2001 Beppu City, Oita, Japan, pp. 544-549.

[11] A. Nasipuri, R. Castaneda and S. Das, "Performance of multipath routing for on-demand protocols inmobile adhoc networks," ACM/Kluwer Mobile Networks and Applications, vol. 6, no. 4, pp. 339-349,2001.

[12] Vincent D. Park and M. Scott Corson, "A Highly Adaptive Distributed Routing Algorithm for Mobile Wireless Networks," Proc. of IEEE INFOCOM '97, Kobe, Japan, April 1997, pp. 1405-1413. 